\title{
Fabrication of Phase-Change Polymer Colloidal Photonic Crystals
}

\author{
Tianyi Zhao, ${ }^{1}$ Youzhuan Zhang, ${ }^{2}$ Jingxia Wang, ${ }^{3}$ Yanlin Song, ${ }^{2}$ and Lei Jiang ${ }^{2}$ \\ ${ }^{1}$ Key Laboratory of Bio-Inspired Smart Interfacial Science and Technology of Ministry of Education, \\ School of Chemistry and Environment, Beihang University, Beijing 100191, China \\ ${ }^{2}$ Beijing National Laboratory for Molecular Sciences (BNLMS), Laboratory of New Materials, \\ Key Laboratory of Organic Solids, Institute of Chemistry, Chinese Academy of Sciences, Beijing 100190, China \\ ${ }^{3}$ Technical Institute of Physics and Chemistry, Chinese Academy of Sciences, Beijing 100190, China \\ Correspondence should be addressed to Jingxia Wang; wangzhang@iccas.ac.cn
}

Received 16 April 2014; Accepted 13 May 2014; Published 12 June 2014

Academic Editor: Yongping Hou

Copyright (C) 2014 Tianyi Zhao et al. This is an open access article distributed under the Creative Commons Attribution License, which permits unrestricted use, distribution, and reproduction in any medium, provided the original work is properly cited.

This paper presents the preparation of phase-change polymer colloidal photonic crystals (PCs) by assembling hollow latex spheres encapsulated with dodecanol for the first time. The monodispersed hollow latex spheres were obtained by phase reversion of monodispersed core-shell latex spheres in the n-hexane, which dissolves the PS core and retains the PMMA/PAA shell. The asprepared phase-change colloidal PCs show stable phase-change behavior. This fabrication of phase-change colloidal PCs would be significant for PC's applications in functional coatings and various optic devices.

\section{Introduction}

Photonic crystals (PCs) have attracted enormous interest due to their unusual optical properties originating from the photonic stopband $[1,2]$ and show potential applications in smart windows, chemical sensors, and optical devices [35]. Recently, increasing interest has been focused on the fabrication of multifunctional PCs; besides their photonic stopband, they can exhibit extra properties and greatly extend their applications [6-12]. For example, tough [13-15] PCs can endure various harsh environments; PCs with special wettability [16-21] favor swift diffusion of the detected materials into the PCs for the highly sensitive sensors, [20] while the responsive PCs [22-25] can respond to various exterior stimuli. Typically, Dorvee et al. [20] reported an amphiphilic, magnetic bifunctional PCs which could manipulate liquid droplets without microfluidic container and its structure color provides an optical signal to distinguish different liquids. It is expected to fabricate novel functional PCs to meet application requirements. Herein, a phase-change polymer colloidal PCs is firstly fabricated by assembly of latex particles encapsulated with phase-change materials (PCMs) of dodecanol, which will greatly extend the applications of PCs in functional coatings.

PCMs [26-29] have attracted wide attention since PCMs can store and release latent heat during their phase transition at defined temperature range, which can be used in thermal energy conservation [29-31]. In the past several decades, research attentions have been mainly paid on the fabrications of phase-change microcapsules $[32,33]$ or phase-change fibers [34], which could retain energy and is used in thermal regulated fabrics, coatings, building, and so forth. It is expected that a novel colloidal PCs could be fabricated if introducing PCMs into the interior of the colloidal PCs. Here, we demonstrate the fabrication of phasechange polymer colloidal PCs by assembly of the hollow latex spheres encapsulated of PCM with n-dodecanol for the first time. The hollow latex spheres are prepared by phase reversion of monodispersed latex spheres with PS core and PMMA/PAA shell in the solvent of $n$-hexane, which is the good solvent of PS core but poor solvent of the PMMA/PAA shell. The as-prepared phase-change colloidal crystals contain $35 \%$ dodecanol, showing a stable phasechange behavior. This fabrication of phase-change PCs with 


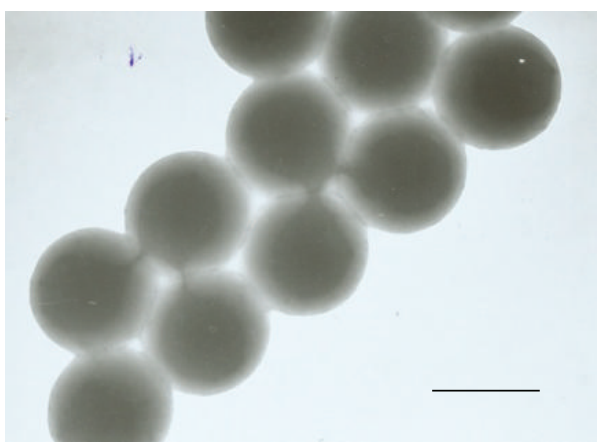

(a)

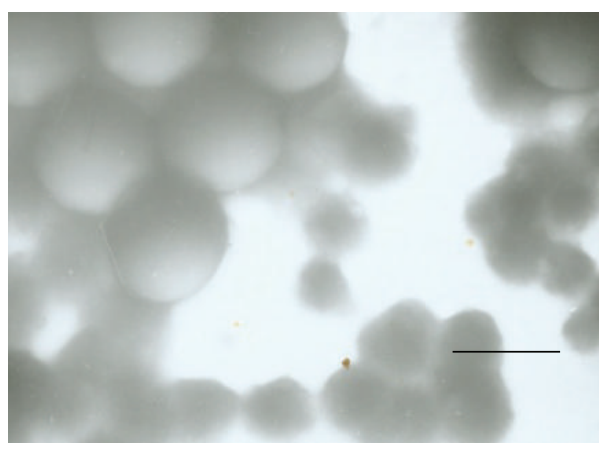

(c)

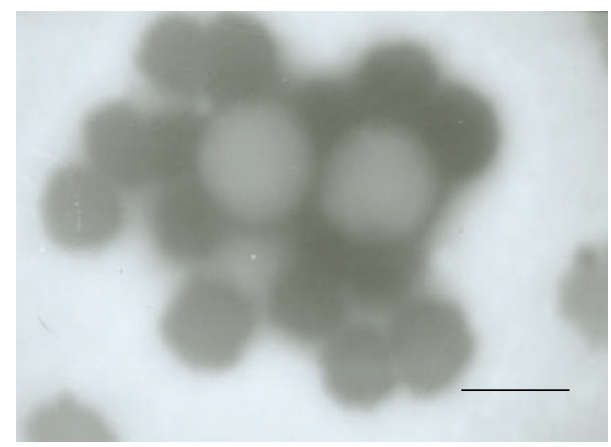

(b)

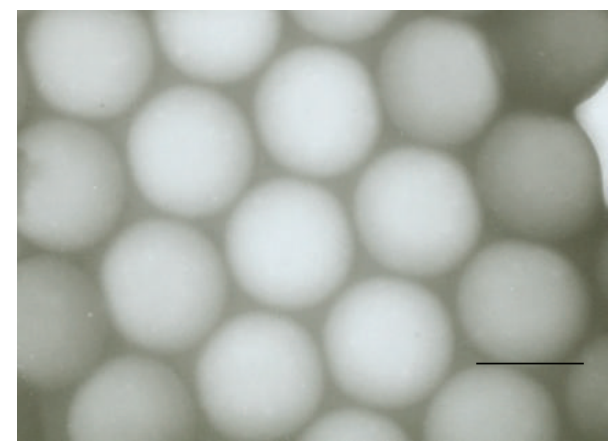

(d)

Figure 1: Typical TEM images of the latex spheres after dispersing in the n-hexane with different times (a) 0, (b) 5, (c) 10, and (d) 30 min. Scale bar: $200 \mathrm{~nm}$.

energy storage properties greatly enlarges the application of polymer colloidal crystals in functional coatings.

\section{Experimental Part}

2.1. Preparation of Monodispersed Latex Spheres. Monodispersed core-shell latex spheres of poly(styrene-methyl methacrylate-acrylic acid) (Poly(St-MMA-AA)) with diameter of $230 \mathrm{~nm}$ were prepared via soap-free emulsion polymerization as reported modified procedure $[15,35]$. The polydispersity of the latex spheres was about 0.005 , which was detected by ZetaPALS BI-90plus (Brookhaven instrument). Glass transition temperature $\left(T_{q}\right)$ of the as-prepared sample was $107.6^{\circ} \mathrm{C}$; the value was analyzed with a Perkin-Elmer DSC 7 (USA).

2.2. Fabrication of Hollow Monodispersed Latex Spheres. The hollow monodispersed latex spheres were prepared as follows [36-38]: firstly, the as-prepared latex suspension was dispersed into the $\mathrm{n}$-hexane $(1: 10, \mathrm{~V} / \mathrm{V})$ by ultrasonic at $30^{\circ} \mathrm{C}$ for $30 \mathrm{~min}$. Secondly, the aforementioned $\mathrm{n}$-hexane phase was removed and the remaining samples were continuously washed via $\mathrm{n}$-hexane for several times. Thus, the hollow latex spheres samples were obtained.

2.3. Filling the Hollow Latex Spheres via n-Dodecanol. The hollow latex spheres were filled with $\mathrm{n}$-dodecanol by dispersing the hollow latex spheres in the solvent of $n$-dodecanol for $30 \mathrm{~min}$ at $30^{\circ} \mathrm{C}$. Subsequently, the filled latex spheres were redispersed into the aqueous system prepared for the use of the self-assembly.

2.4. Fabrication of Phase-Change Colloidal PCs. The phasechange colloidal PCs were fabricated on glass substrates by vertical deposition method $[15,35]$. Glass slides were treated firstly with a chromic-sulfuric acid solution to ensure clean surfaces and then fixed vertically in a vial containing the monodisperse hollow PMMA-PAA aqueous suspension of $0.2 \mathrm{wt} \%$ at constant temperature $\left(25^{\circ} \mathrm{C}\right)$ and humidity $(60$ R.H.\%) for $24 \mathrm{~h}$. Then, the dried phase-change PCs were obtained.

2.5. Characterization. Transmission electron microscopy (TEM) images of latex spheres were obtained with a H800 transmission microscope (JEM2010, Japan); high resolution TEM (HRTEM) (Tecnai F30) was used to investigate the latex morphology. Scanning electron microscopy (SEM) images were obtained with a field-emission scanning electron microscope (JEOL JSM-6700, Japan). UV-Vis spectra were performed on a U-4100 UV-Vis Spectrometer (Hitachi, Japan). A Nicolet Magna 750 FTIR spectrometer was used to investigate the chemical component of the colloidal crystal films by attenuated total reflectance infrared spectroscopy (ATRFTIR) mode. The phase-change property of the samples was determined by DSC (Shimadzu DSC-60) in Al pans with a scanning rate of $4^{\circ} \mathrm{C} / \mathrm{min}$. 


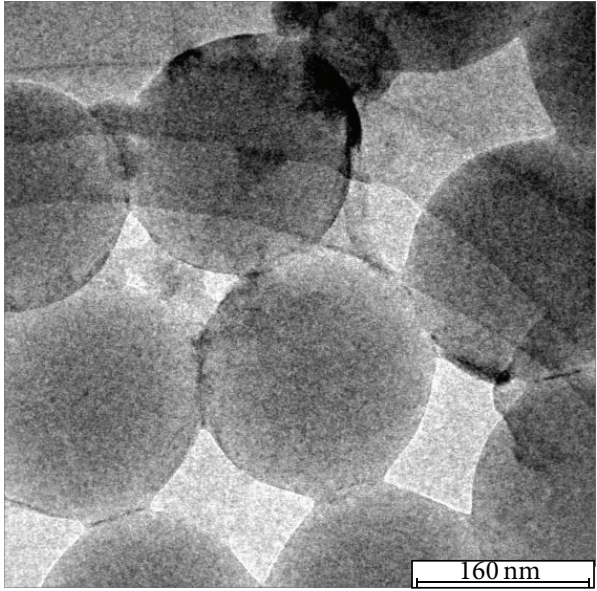

(a)

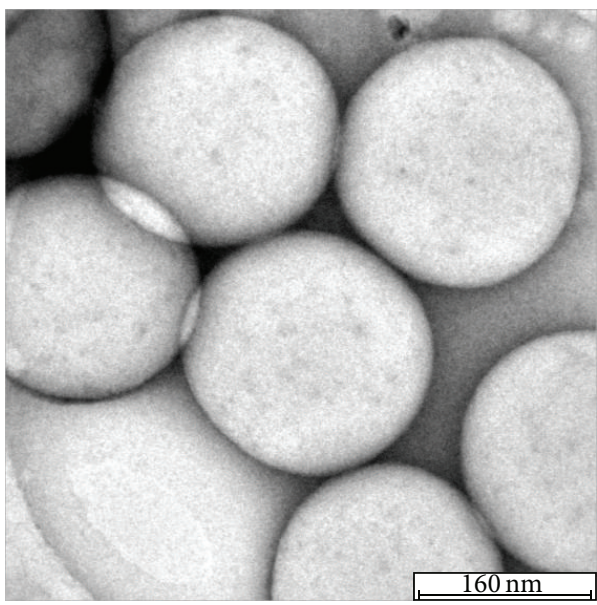

(c)

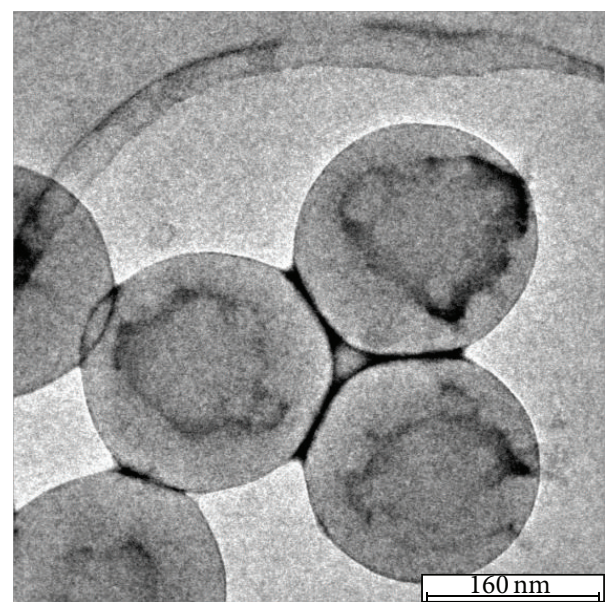

(b)

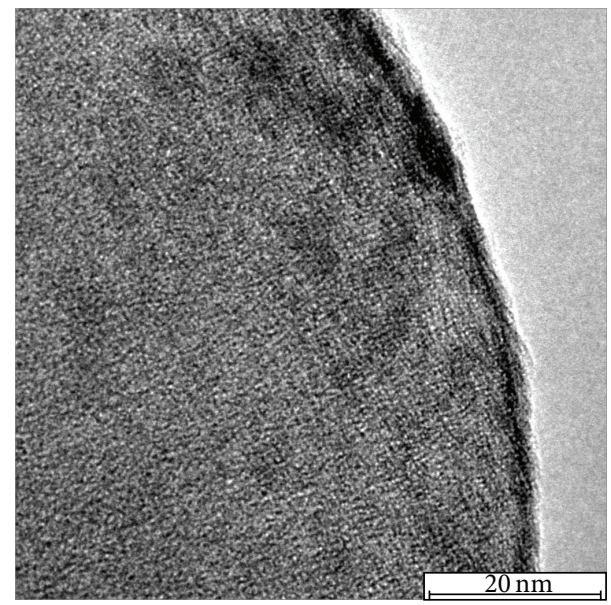

(d)

FIGURE 2: HRTEM images of the films. (a) Solid spheres, (b) latex spheres dispersing in $\mathrm{n}$-hexane for 5 min, (c) hollow latex spheres, and (d) high-magnification image of the hollow sphere. Scale bar from (a) to (c) is $160 \mathrm{~nm}$ and scale bar of (d) is $20 \mathrm{~nm}$.

\section{Results and Discussion}

The monodispersed latex spheres with hydrophobic PS core and hydrophilic PMMA/PAA shell were prepared via onestep soap-free emulsion polymerization, $[15,35,39]$ where hydrophilic AA and hydrophobic St were dispersed in the aqueous phase and the spontaneous formation of hydrophilic shell and hydrophobic core was mainly attributed to the minimization of the total interfacial energy in the aqueous phase [39]. Thus, hydrophobic polymer segment (PS-rich) would try to depart from water phase, while the hydrophilic polymer segment (PMMA/PAA-rich) would prefer to extend toward the water phase. As a consequence, the latex spheres with PS-rich core and PMMA/PAA-rich shell were formed. The core-shell morphology can be clearly confirmed from TEM images of Figure 1(a), where, the darkly PS-rich regions are surrounded by the grey PAA/PMMA-rich domains.

The hollow latex spheres are prepared by dispersing the as-prepared latex suspension with PS core-PMMA/PAA shell into the solvent of n-hexane, which is a good solvent of PS core but poor solvent of PMMA/PAA shell. Figures 1(a)1(d) present TEM images of the latex suspension with PS core-PMMA/PAA shell after being dispersed in $n$-hexane for different times. Clearly, the core part of latex spheres gradually decreases and disappears when dispersing the latex spheres in the n-hexane for 5, 10, and $30 \mathrm{~min}$, which indicates that the hollow latex sphere can be obtained via the abovementioned process. In details, the black hydrophobic PS core will effuse and melt into the $n$-hexane phase once the latex spheres are dispersed into the n-hexane in Figure 1(b), which is very different from that before the sample is dispersed into the n-hexane; latex particles keep distinct black hydrophobic core and grey hydrophilic shell in Figure 1(a). The irregular black particle in Figures 1(b) and 1(c) is the melted PS core since the black hydrophobic PS-rich core tends to extend toward the hydrophobic n-hexane solvent phase due to their similar solubility and phase separation [36-38]. With the increased ultrasonic time, most of PS-rich segment in the core dissolves into the $\mathrm{n}$-hexane. As a result, the insoluble PMMA/PAA-rich segment is retained and the latex sphere 


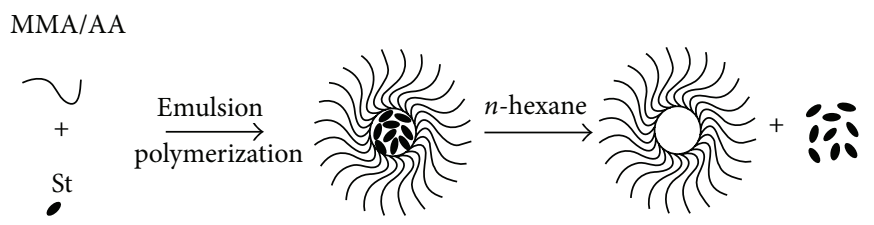

(a)

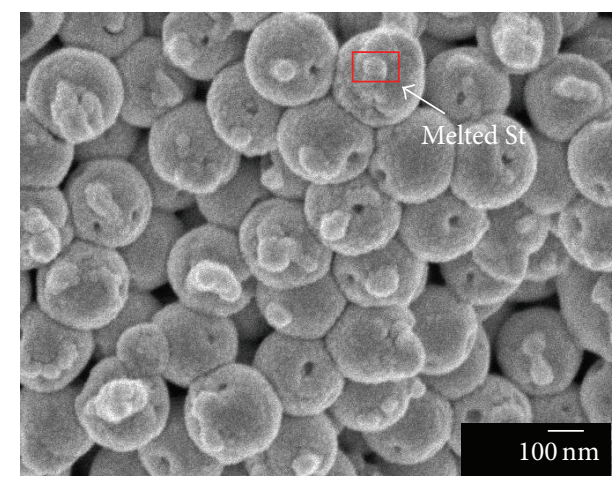

(b)

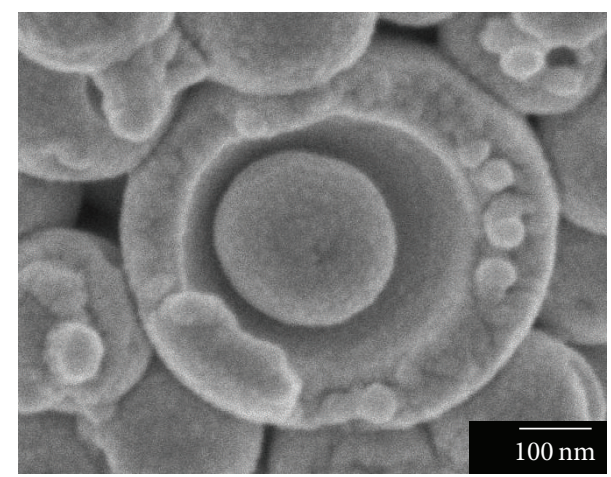

(c)

FIGURE 3: (a) Schematic illustration of the formation process from solid sphere to hollow sphere. (b) and (c) SEM images of the films assembled from latex spheres dispersing in $\mathrm{n}$-hexane for $5 \mathrm{~min}$.

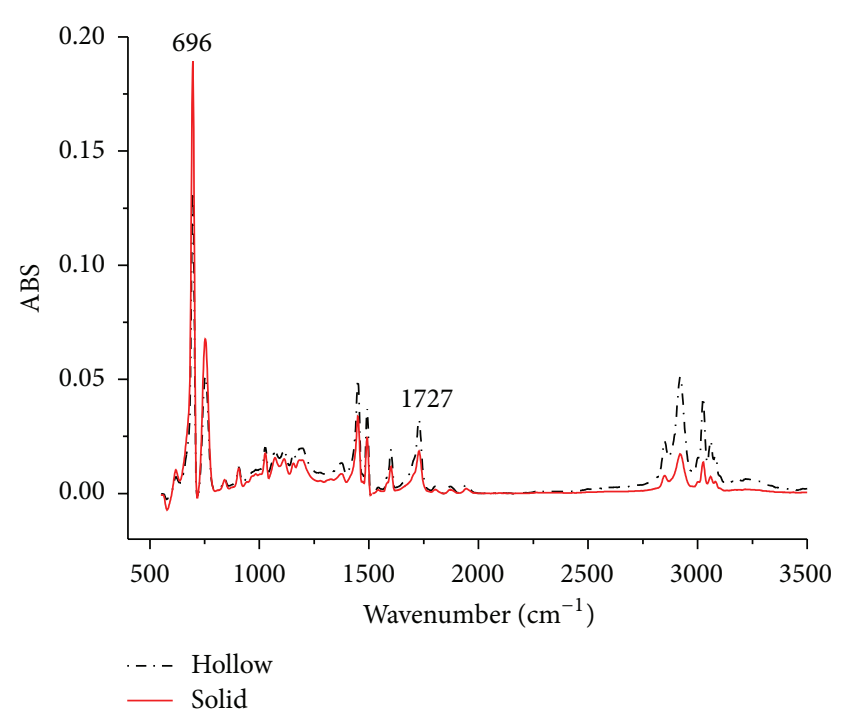

FIGURE 4: ATR FTIR spectra of solid sphere (red solid line) and hollow sphere (black dot line).

keeps the spherical shape, which results in the formation of hollow latex spheres made of PMMA/PAA in Figures 1(c) and $1(\mathrm{~d})$. But the broken latex spheres would be observed if ultrasonic time is over $30 \mathrm{~min}$. High magnification HRTEM images in Figure 2 confirm the elimination of the PS core and the achievement of the hollow P(MMA-AA) latex spheres. However, no clear cavity could be observed in the shell of hollow latex sphere, as given in the magnified HRTEM image in Figure 2(d). But an obvious cavity could be found in the latex particle from the SEM pictures, which confirms the obtainment of the latex particle with hollow structure when further putting the hollow latex particles in the liquid $\mathrm{N}_{2}$ for 5 min (see Figure S1, see supplementary material available online at http://dx.doi.org/10.1155/2014/702089).

Furthermore, the influence of the mixing ratio of $n$ hexane and polymer on the resultant morphology of latex spheres has been optimized (see Table S1). When the volume ratio of the latex and $n$-hexane is smaller than $1: 1$, the dissolution effect is insufficient for the removement of PS core. But the PS residue is found when the ratio of $n$-hexane is smaller than 10 and the broken latex spheres will be observed when the solvent ratio is larger than 10. It is found that the optimized volume ratio of the latex and n-hexane is $1: 10$. The possible formation procedure of the hollow latex spheres was also presented in the upper part of Figure 3 (Figure 3(a)). The initial core-shell latex spheres with PSrich core and PMMA/PAA-rich shell are firstly synthesized by one-step emulsion polymerization. When the as-prepared latex spheres are dispersed into the solvent of n-hexane, phase reversion occurs to the polymer segments with different polarity. As a result, the PS-rich polymer segment in the latex core would be dissolved out while the PMMA/PAArich polymer shell would keep intact. The procedure can be further elaborated by the SEM images of the film assembled from latex spheres which were just undergone the process of dispersing into the solvent. From Figure 3(b), it can be clearly observed that PS residue formed irregular particles onto the PMMA/PAA polymer shells when dispersing into the nhexane solvent. From the magnified image in Figure 3(c), it can also be observed that core part of the latex sphere was reduced because of the dispersing process.

To confirm clearly the dissolution or part dissolution of PS-rich core in n-hexane solvent, ATR $[19,40]$ spectra is used to check the change of the chemical composition of the films 


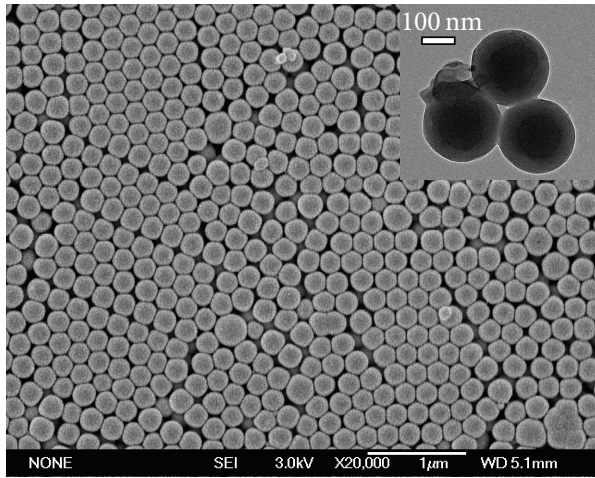

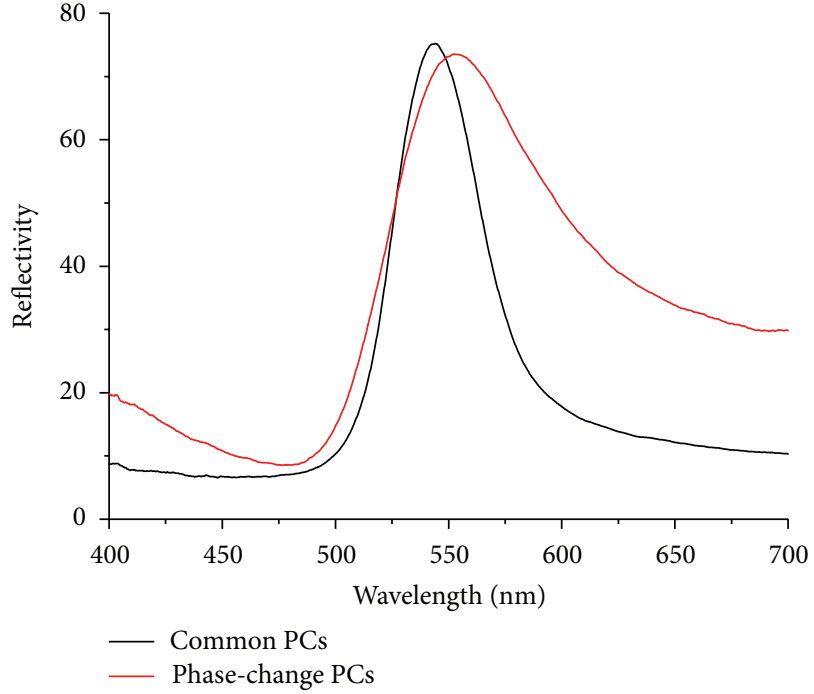

(b)

FIGURE 5: (a) SEM images and (b) UV-Vis spectra of the as-prepared phase-change and common PCs; the insert is the TEM image of the latex particle encapsulated with n-dedocanol.

assembled from latex spheres before and after being dispersed in the solvent. The result is demonstrated in Figure 4. In this experiment, the change of chemical component of the colloidal crystal films is estimated from the different absorbance of specific infrared bands, in particular, the change of absorbance ratios between PS and PMMA/PAA. In this case, the characteristic absorbance bands of $1727 \mathrm{~cm}^{-1}$ from carboxyl group, and $696 \mathrm{~cm}^{-1}$ from benzene group, represent the chemical composition of core (PS) and shell (PMMA/PAA) part [19]. As an example, Figure 4 presents the ATR spectra of the colloidal crystal films assembled from hollow or solid latex spheres, respectively. And the absorbance ratio between the absorbance bands 696 and $1727 \mathrm{~cm}^{-1}$ is 9.88 before the latex spheres are dispersed into the solvent and the value lowers and reaches 3.93 after dispersing into the solution. The result indicates the decrease of the ratio of PS segment after dispersing the latex spheres in the solution; the characterization result is well agreed with the phase reversion or disappearance of the PS-rich core part in Figure 1.

The phase-change colloidal PCs were obtained by assembly of hollow latex spheres filled with $n$-dodecanol. The insert picture of Figure 5(a) is TEM image of the latex particle infiltrated in $\mathrm{n}$-dodecanol, the clear flow of the dodecanol could be found in magnified image in Figure S2. Figure 5 shows the SEM image and the UV-vis spectra of the asprepared phase-change colloidal PCs. Clearly, there is wellordered arrangement for the latex spheres in the films in Figure 5(a), which contributes to the good optic properties in Figure 5(b). But the reflection spectra of phase-change colloidal PCs is broader than that of PCs from common latex particles, which indicates that the dissolution process of core may result in some deformation of latex spheres. This affects the assembly and optic properties of the resultant film.

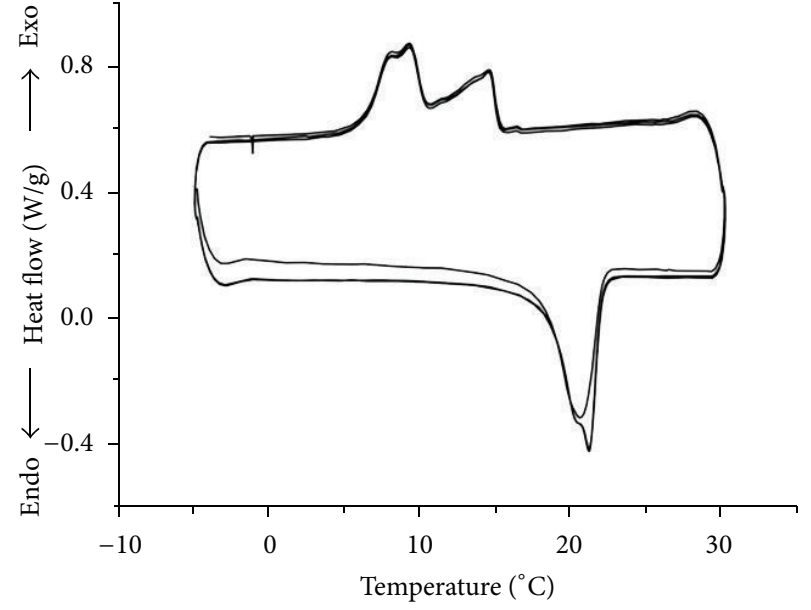

FIGURE 6: DSC measurement of the as-prepared phase-change polymer colloidal PCs. The phase-change material is n-dedocanol.

Phase-change properties of the resultant colloidal PCs could be clearly seen from DSC measurement in Lissajous figures of Figure 6. Because the melting point of the ndodecanol is about $24^{\circ} \mathrm{C}$, we characterize the thermal properties of as-prepared phase-change colloidal PCs ranging from 0 to $30^{\circ} \mathrm{C}$ with a heating/cooling rate of $4^{\circ} \mathrm{C} / \mathrm{min}$ for three cycles. The as-prepared colloidal PCs have two obvious absorb and release processes which correspond to the melting point and freezing point of the n-dodecanol, respectively. Among these peaks, the melting point $\left(21.23^{\circ} \mathrm{C}\right)$ and the freezing point $\left(8\right.$ and $\left.14^{\circ} \mathrm{C}\right)$ indicate the existence of the $n$ dodecanol in the as-prepared colloidal PCs. It should be noted that two exothermic peaks occur during the cooling process, which may be attributed to the influence of the latex 
polymer on bulk crystallization, which leads to the lower enthalpy than that of melt peak. The measured enthalpy of melting of the sample was about $14.01 \mathrm{~J} / \mathrm{g}$; it could be calculated that about $35 \% \mathrm{n}$-dodecanol is encapsulated into the latex particle. More importantly, the good repeatability of cycle measurement indicates that the PCMs have been encapsulated independently in the latex spheres without leakage.

\section{Conclusion}

The paper demonstrated a facile fabrication of hollow latex spheres from phase separation of core-shell latex spheres and the hollow latex spheres were encapsulated with n-dedecanol. The phase-change polymer colloidal crystals assembled from latex spheres filled with n-dodecanol showed stable phasechange behavior. The fabrication of phase-change colloidal PCs is significant for the promising application in functional coatings.

\section{Conflict of Interests}

The authors declare that there is no conflict of interests regarding the publication of this paper.

\section{Acknowledgments}

This work is supported by National Natural Science Foundation of China (nos. 21101010, 91127029, 51373183, and 21074139) and 973 Program (nos. 2011CB932303 and 2011CB8084004).

\section{References}

[1] Y. Huang, J. Zhou, B. Su et al., "Colloidal photonic crystals with narrow stopbands assembled from low-adhesive superhydrophobic substrates," Journal of the American Chemical Society, vol. 134, no. 41, pp. 17053-17058, 2012.

[2] R. Wagner and F. Cichos, "Fast measurement of photonic stop bands by back focal plane imaging," Physical Review B: Condensed Matter and Materials Physics, vol. 87, no. 16, Article ID 165438, 2013.

[3] X. Huang, Y. Lai, Z. H. Hang, H. Zheng, and C. T. Chan, "Dirac cones induced by accidental degeneracy in photonic crystals and zero-refractive-index materials," Nature Materials, vol. 10, no. 8, pp. 582-586, 2011.

[4] A. Mihi, C. Zhang, and P. V. Braun, "Transfer of preformed three, dimensional photonic crystals onto dye, sensitized solar cells," Angewandte Chemie, vol. 123, pp. 5830-5833, 2011.

[5] C. I. Aguirre, E. Reguera, and A. Stein, "Tunable colors in opals and inverse opal photonic crystals," Advanced Functional Materials, vol. 20, no. 16, pp. 2565-2578, 2010.

[6] C. Zhu, L. Chen, H. Xu, and Z. Gu, "A magnetically tunable colloidal crystal film for reflective display," Macromolecular Rapid Communications, vol. 30, no. 22, pp. 1945-1949, 2009.

[7] H. Wang, Q.-W. Chen, Y.-F. Yu, K. Cheng, and Y.-B. Sun, "Sizeand solvent-dependent magnetically responsive optical diffraction of carbon-encapsulated superparamagnetic colloidal photonic crystals," Journal of Physical Chemistry C, vol. 115, no. 23, pp. 11427-11434, 2011.
[8] A. Ghadimi, L. Cademartiri, U. Kamp, and G. A. Ozin, "Plasma within templates: molding flexible nanocrystal solids into multifunctional architectures," Nano Letters, vol. 7, no. 12, pp. 38643868, 2007.

[9] M. E. Calvo, S. Colodrero, N. Hidalgo et al., "Porous one dimensional photonic crystals: novel multifunctional materials for environmental and energy applications," Energy and Environmental Science, vol. 4, no. 12, pp. 4800-4812, 2011.

[10] C.-Y. Liu and L.-W. Chen, “Tunable channel drop filter in a twodimensional photonic crystal modulated by a nematic liquid crystal," Journal of Nanomaterials, vol. 2006, Article ID 52946, 6 pages, 2006.

[11] J. Riedrich-Möller, L. Kipfstuhl, C. Hepp et al., "One-and twodimensional photonic crystal microcavities in single crystal diamond," Nature Nanotechnology, vol. 7, no. 1, pp. 69-74, 2012.

[12] P. Alivisatos, "The use of nanocrystals in biological detection," Nature Biotechnology, vol. 22, no. 1, pp. 47-52, 2004.

[13] B. You, N. Wen, L. Shi, L. Wu, and J. Zi, "Facile fabrication of a three-dimensional colloidal crystal film with large-area and robust mechanical properties," Journal of Materials Chemistry, vol. 19, no. 22, pp. 3594-3597, 2009.

[14] J. Zhou, H. Li, L. Ye et al., "Facile fabrication of tough SiC inverse opal photonic crystals," Journal of Physical Chemistry C, vol. 114, no. 50, pp. 22303-22308, 2010.

[15] J. Wang, Y. Wen, H. Ge et al., "Simple fabrication of full color colloidal crystal films with tough mechanical strength," Macromolecular Chemistry and Physics, vol. 207, no. 6, pp. 596604, 2006.

[16] J. Wang, Y. Zhang, S. Wang, Y. Song, and L. Jiang, "Bioinspired colloidal photonic crystals with controllable wettability," Accounts of Chemical Research, vol. 44, no. 6, pp. 405-415, 2011.

[17] L. Cui, Y. Li, J. Wang et al., "Fabrication of large-area patterned photonic crystals by ink-jet printing," Journal of Materials Chemistry, vol. 19, no. 31, pp. 5499-5502, 2009.

[18] O. Sato, S. Kubo, and G. U. Zhong-Ze, "Structural color films with lotus effects, superhydrophilicity, and tunable stop-bands," Accounts of Chemical Research, vol. 42, no. 1, pp. 1-10, 2009.

[19] J. Wang, Y. Wen, J. Hu, Y. Song, and L. Jiang, "Fine control of the wettability transition temperature of colloidal-crystal films: from superhydrophilic to superhydrophobic," Advanced Functional Materials, vol. 17, no. 2, pp. 219-225, 2007.

[20] J. R. Dorvee, A. M. Derfus, S. N. Bhatia, and M. J. Sailor, "Manipulation of liquid droplets using amphiphilic, magnetic one-dimensional photonic crystal chaperones," Nature Materials, vol. 3, no. 12, pp. 896-899, 2004.

[21] M. Kuang, J. Wang, B. Bao et al., "Inkjet printing patterned photonic crystal domes for wide viewing, angle displays by controlling the sliding three phase contact line," Advanced Optical Materials, vol. 2, no. 1, pp. 34-38, 2014.

[22] G. Jianping, H. Le, J. Goebl, and Y. Yin, "Assembly of magnetically tunable photonic crystals in nonpolar solvents," Journal of the American Chemical Society, vol. 131, no. 10, pp. 3484-3486, 2009.

[23] X. Xu, S. A. Majetich, and S. A. Asher, "Mesoscopic monodisperse ferromagnetic colloids enable magnetically controlled photonic crystals," Journal of the American Chemical Society, vol. 124, no. 46, pp. 13864-13868, 2002.

[24] J. Ge and Y. Yin, "Responsive photonic crystals," Angewandte Chemie, vol. 50, no. 7, pp. 1492-1522, 2011.

[25] H. Wang, Y.-B. Sun, Q.-W. Chen, Y.-F. Yu, and K. Cheng, "Synthesis of carbon-encapsulated superparamagnetic colloidal 
nanoparticles with magnetic-responsive photonic crystal property," Dalton Transactions, vol. 39, no. 40, pp. 9565-9569, 2010.

[26] S. Raoux and M. Wuttig, Phase Change Materials, Springer, 2009.

[27] M. Wuttig and N. Yamada, "Phase-change materials for rewriteable data storage," Nature Materials, vol. 6, no. 11, pp. 824-832, 2007.

[28] M. Wuttig, "Phase-change materials: towards a universal memory?” Nature Materials, vol. 4, no. 4, pp. 265-266, 2005.

[29] A. Sharma, V. V. Tyagi, C. R. Chen, and D. Buddhi, "Review on thermal energy storage with phase change materials and applications," Renewable and Sustainable Energy Reviews, vol. 13, no. 2, pp. 318-345, 2009.

[30] L. F. Cabeza, A. Castell, C. Barreneche, A. De Gracia, and A. I. Fernández, "Materials used as PCM in thermal energy storage in buildings: a review," Renewable and Sustainable Energy Reviews, vol. 15, no. 3, pp. 1675-1695, 2011.

[31] D. Zhou, C. Y. Zhao, and Y. Tian, "Review on thermal energy storage with phase change materials (PCMs) in building applications," Applied Energy, vol. 92, pp. 593-605, 2012.

[32] L. Bayés-García, L. Ventolà, R. Cordobilla, R. Benages, T. Calvet, and M. A. Cuevas-Diarte, "Phase Change Materials (PCM) microcapsules with different shell compositions: preparation, characterization and thermal stability," Solar Energy Materials and Solar Cells, vol. 94, no. 7, pp. 1235-1240, 2010.

[33] C. Y. Zhao and G. H. Zhang, "Review on microencapsulated phase change materials (MEPCMs): fabrication, characterization and applications," Renewable and Sustainable Energy Reviews, vol. 15, no. 8, pp. 3813-3832, 2011.

[34] S.-H. Lee, Y. Jung, and R. Agarwal, "Highly scalable non-volatile and ultra-low-power phase-change nanowire memory," Nature Nanotechnology, vol. 2, no. 10, pp. 626-630, 2007.

[35] J. Wang, Y. Wen, X. Feng, Y. Song, and L. Jiang, "Control over the wettability of colloidal crystal films by assembly temperature," Macromolecular Rapid Communications, vol. 27, no. 3, pp. 188192, 2006.

[36] J. Wang and M. Jiang, "Polymeric self-assembly into micelles and hollow spheres with multiscale cavities driven by inclusion complexation," Journal of the American Chemical Society, vol. 128, no. 11, pp. 3703-3708, 2006.

[37] Q. Zhang, E. E. Remsen, and K. L. Wooley, "Shell cross-linked nanoparticles containing hydrolyrically degradable, crystalline core domains," Journal of the American Chemical Society, vol. 122, no. 15, pp. 3642-3651, 2000.

[38] J. Fu, J. Wang, Q. Li, D. H. Kim, and W. Knoll, “3D hierarchically ordered composite block copolymer hollow sphere arrays by solution wetting," Langmuir, vol. 26, no. 14, pp. 12336-12341, 2010.

[39] F. Hoshino, H. Kawaguchi, and Y. Ohtsuka, "N-Substituted acrylamide-styrene copolymer latices. III: morphology of latex particles," Polymer Journal, vol. 19, no. 10, pp. 1157-1164, 1987.

[40] Y. Liang and H. S. Lee, "Surface-induced crystal orientation of poly(trimethylene 2,6-naphthalate) films studied by polarized FTIR-ATR and grazing incidence X-ray diffraction," Macromolecules, vol. 38, no. 23, pp. 9885-9888, 2005. 

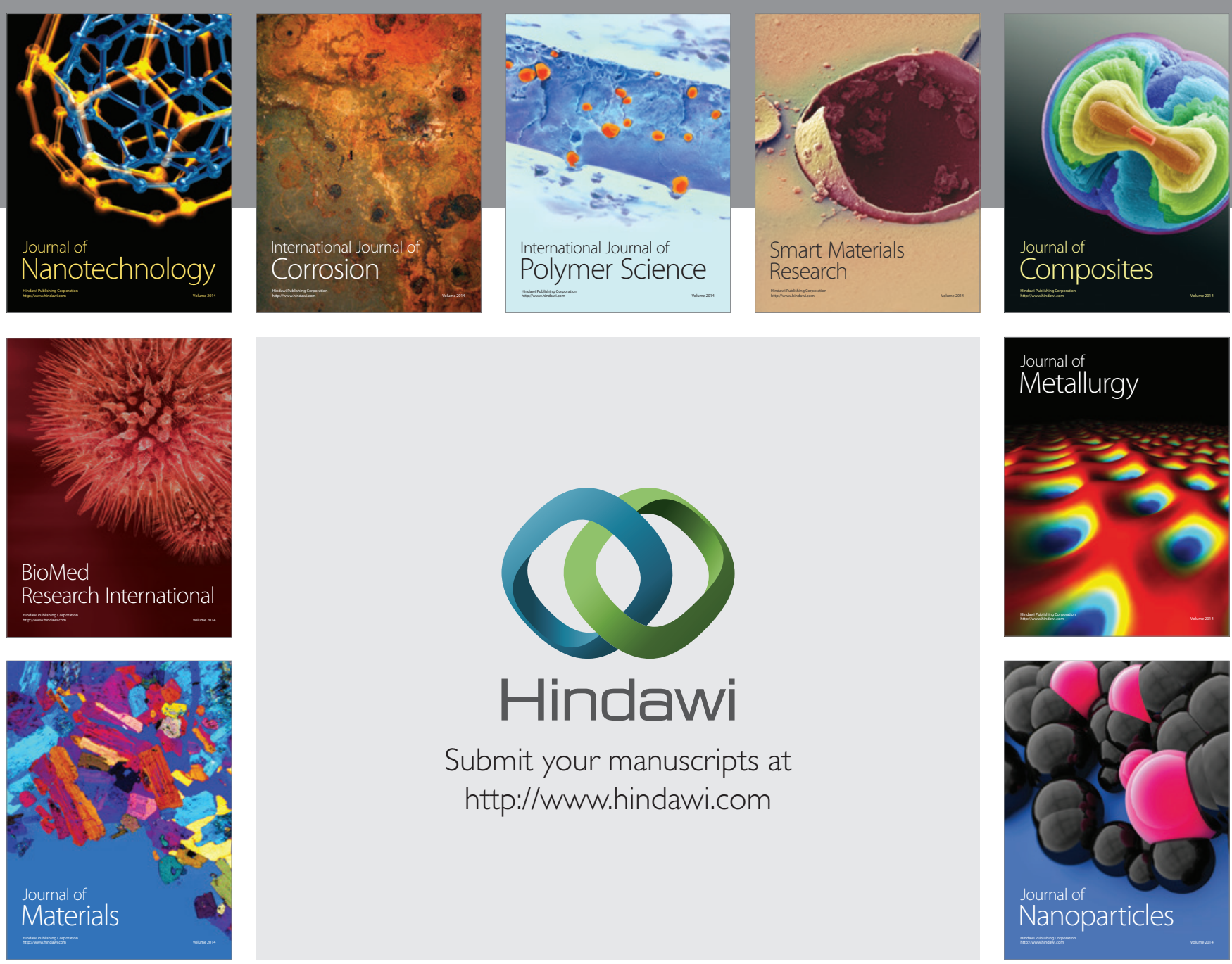

Submit your manuscripts at http://www.hindawi.com
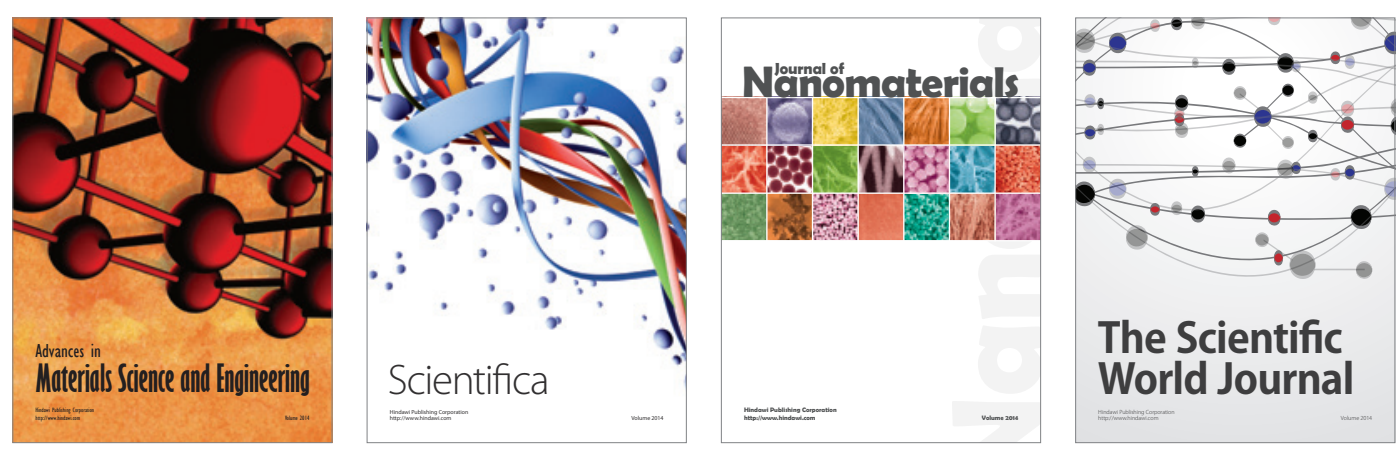

\section{The Scientific World Journal}
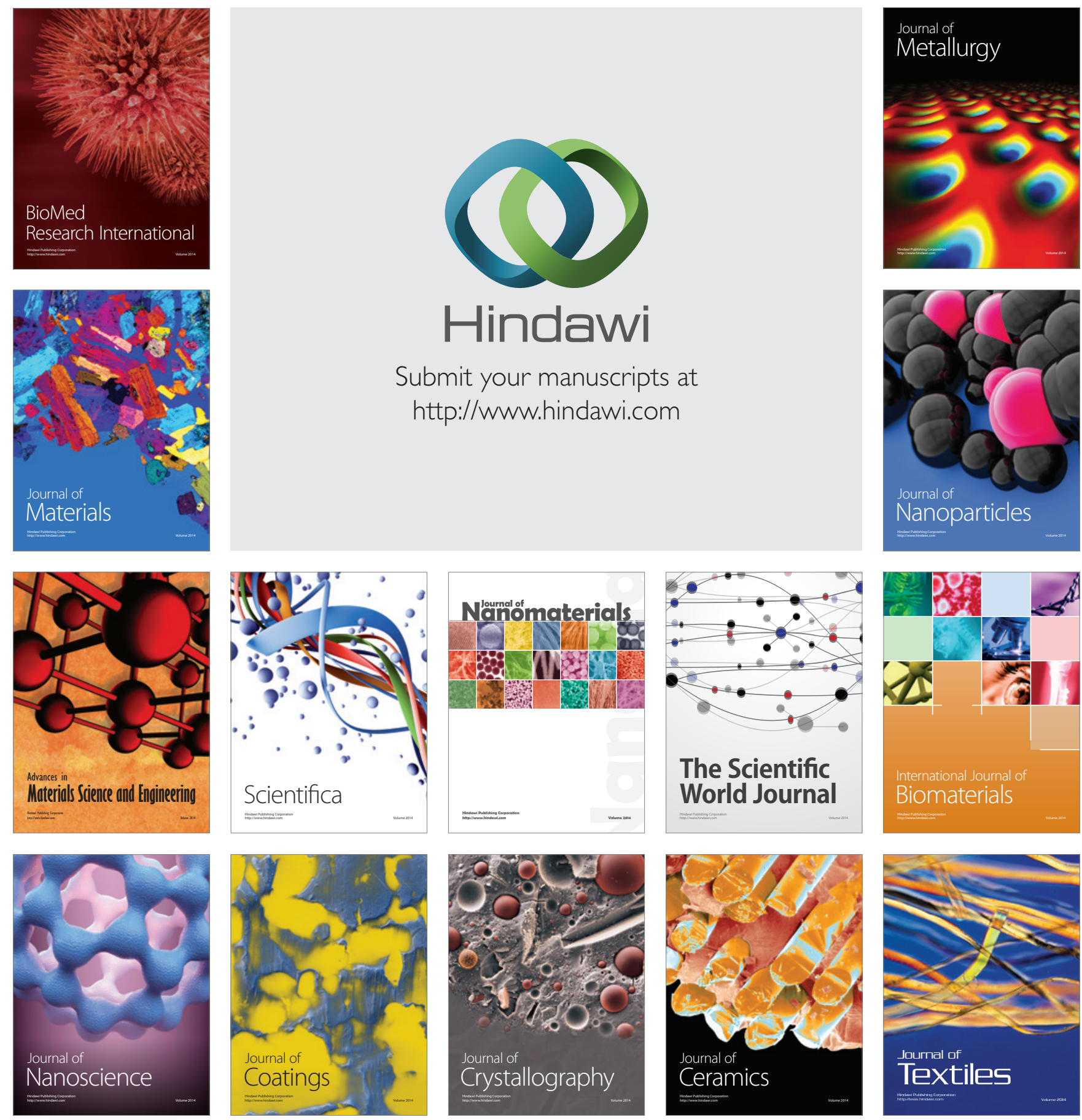